\title{
Ferramentas educacionais digitais para crianças autistas
}

\author{
Digital educational tools for autistic children \\ Herramientas educativas digitales para niños autisticos
}

Recebido: 28/07/2021 | Revisado: 03/08/2021 | Aceito: 04/08/2021 | Publicado: 09/08/2021

\author{
Ricardo Sousa Cavalcanti \\ ORCID: https://orcid.org/0000-0002-7348-8946 \\ Instituto Federal de Educação, Ciência e Tecnologia de Minas Gerais, Brasil \\ E-mail: ricardo.cavalcanti@ifmg.edu.br \\ Lílian Amaral de Carvalho \\ ORCID: http://orcid.org/0000-0001-6672-654X \\ Instituto Federal de Educação, Ciência e Tecnologia de Minas Gerais, Brasil \\ E-mail: lilian.carvalho@ifmg.edu.br
}

\begin{abstract}
Resumo
Os avanços tecnológicos buscam trazer melhorias na vida das pessoas, principalmente com o atual advento da inteligência artificial. Essa tecnologia tem buscado auxiliar a vida das crianças com Transtorno do Espectro Autista (TEA) e de seus pais, melhorando a qualidade de vida e a interação com a sociedade em que vivem. Este trabalho foi realizado a partir de uma revisão bibliográfica em periódicos científicos de forma a apresentar alguns aplicativos destinados às crianças com TEA para a promoção da interação delas com o ambiente social em que vivem. A revisão bibliográfica deu ênfase aos aplicativos que apresentam idioma em língua portuguesa, ainda estão disponíveis para download e preferencialmente gratuitos para crianças com TEA. Foram selecionados e descritos 14 aplicativos, adicionando-se algumas avaliações dos benefícios e dificuldades quando elas eram apresentadas, além de mostrar figuras representativas e os endereços eletrônicos para acesso aos mesmos. O conhecimento desses aplicativos é de suma importância para os responsáveis e para a equipe clínica que trabalha com pessoas com TEA e pode contribuir para um desenvolvimento mais rápido e para a melhoria da qualidade de vida das pessoas com TEA.
\end{abstract}

Palavras-chave: Transtorno do Espectro Autista; Aplicativos; Ensino; Educação Inclusiva.

\begin{abstract}
Technological advances look improvements in people's lives, especially with the current advent of artificial intelligence. This technology has sought to help the lives of children with Autistic Spectrum Disorder (ASD) and their parents, improving their quality of life and interaction with the society in which they live. This work was carried out from a literature review in scientific journals in order to present some applications for help children with ASD to promote their interaction with the social environment in which they live. The bibliographical review emphasized the applications that present the language in Portuguese, are still available for download and preferably free for help in treatment of children with ASD. Aproximatly14 applications were selected and described, adding some assessments of the benefits and difficulties when they were presented, in addition to showing representative figures and the electronic addresses to access them. Knowledge of these applications is of paramount importance for those responsible and for the clinical team that works with people with ASD and can contribute to a faster development and to improve the quality of life of people with ASD.
\end{abstract}

Keywords: Autistic Spectrum Disorder; Applications; Teaching; Inclusive Education.

\section{Resumen}

Los avances tecnológicos buscan mejorar la vida de las personas, especialmente con el avance actual de la inteligencia artificial. Esta tecnología ha buscado ayudar la vida de los niños con Trastorno del Espectro Autista (TEA) y sus padres, mejorando su calidad de vida e interacción con la sociedad en la que viven. Este trabajo se realizó a partir de una revisión de la literatura en revistas científicas con el fin de presentar algunas aplicaciones para niños con TEA para promover su interacción con el entorno social en el que viven. La revisión bibliográfica destacó las aplicaciones que presentan en idioma portugués, todavía están disponibles para descargar y preferiblemente gratis como soporte para niños con TEA. Se seleccionaron y describieron aproximadamente 14 aplicaciones, agregando algunas valoraciones de los beneficios y dificultades cuando se presentaron, además de mostrar cifras representativas y las direcciones electrónicas para acceder a ellas. El conocimiento de estas aplicaciones es de suma importancia para los responsables y para el equipo clínico que trabaja con personas con TEA y puede contribuir a un desarrollo más rápido y a mejorar la calidad de vida de las personas con TEA.

Palabras clave: Trastorno del Espectro Autista; Aplicaciones, Enseñanza; Educación Inclusiva. 


\section{Introdução}

A tecnologia lida com o uso humano e o conhecimento de instrumentos e técnicas para ajudar as pessoas a controlar e se adaptar ao ambiente. Não poderia ser diferente para os indivíduos com o Transtorno do Espectro Autista (TEA), em que o uso da tecnologia está assumindo um papel cada vez mais importante na pesquisa e na prática clínica relacionada às crianças com esse tipo de transtorno.

A evolução tecnológica caminha na direção de tornar a vida das pessoas mais fácil. Um exemplo é a tecnologia assistiva, que está sendo amplamente utilizada em conjunto com dispositivos móveis devido às suas vantagens de mobilidade, acesso à informação de forma rápida e de qualquer lugar, tornando um recurso eficaz no atendimento de necessidades específicas. Isso tem proporcionado, à pessoa que apresneta alguma dificuldade, uma maior independência, qualidade de vida e inclusão social, levando a novas e mais eficazes estratégias de tratamento e melhorando a qualidade de vida para os usuários e seus familiares (Bölte, Goodwin, \& Waigenbaum, 2010; Lucian \& Stumpf, 2019).

As crianças com TEA foram amparadas pela Lei $\mathrm{n}^{\circ}$ 12.764/12, nos artigos 1 e 2, somente a partir de 2012, sendo reconhecidas como pessoas com deficiência, em que o termo AUTISMO têm como significado estar-se ausente ou perdido, caracterizando-as pelo distanciamento da realidade que cerca a pessoa com TEA, podendo apresentar padrões de comportamentos diferentes ou não, havendo casos em que se mostram bastante diferenciados dos demais (Brasil, 2012). Esse agir incomum que o afasta da "realidade normal" no ambiente escolar pode causar dificuldades no seu processo de interação social e acarretar consequências na aprendizagem, assimilação e acomodação dos conteúdos (Silva \& Silva, 2016).

O transtorno do espectro autista é um transtorno do neurodesenvolvimento que, por causa dos principais sintomas de deficiência na comunicação e na interação social, além do comportamento repetitivo, muitas vezes compromete seriamente a adaptação e a inclusão social. As deficiências de pacientes com TEA são geralmente tratadas através de reabilitação com auxílio de terapeutas, o que torna o seu tratamento muito oneroso (Brasil, 2012; Lucian \& Stumpf, 2019).

Para facilitar a interação dos alunos com TEA, os professores e tutores utilizam estratégias educacionais e auxiliam os pais a interagirem com o(a) filho(a) autista, o que promove o desenvolvimento de competências desses alunos. O convívio e a troca de experiências entre a família e escola são essenciais para a formação pessoal e profissional da criança, pois servirão de suporte para o desenvolvimento intelectual do aluno autista (Souza, Silva, \& Silva, 2019).

Como foi citado, as crianças com TEA exigem uma tarefa muito desafiadora de seus pais e, também, dos professores, por causa de suas dificuldades em expressar suas necessidades verbalmente. Elas costumam usar gestos, como apontar, ao invés de falar. Alguns aplicativos têm sido indicados para preencher esta lacuna, auxiliando as crianças a se comunicarem e docentes e pais nessa desafiadora tarefa de inclusão. Outras ações também têm contribuído para a inclusão desses alunos, como, por exemplo, alguns educadores têm utilizado atividades didáticas que instigam a curiosidade pelo uso da ludicidade com materiais concretos, que atraem a atenção dos alunos e trabalham a coletividade entre as crianças (Lucian \& Stumpf, 2019; Souza et al., 2019; Silva, Oliveira, Campos \& Oliveira, 2019).

Os aplicativos, também conhecidos como APPs, são ferramentas tecnológicas desenvolvidas principalmente para dispositivos móveis, como smartphones, tablets, entre outros. Eles possuem variadas funções para os usuários, incluindo tecnologias educacionais que ajudam os pais a inserirem seus filhos autistas nos ambientes sociais e escolares (Lucian \& Stumpf, 2019; Aragão, Bottentuit Júnior, \& Zaqueu, 2019).

Segundo Lucian e Stumpf (2019) é possível encontrar diversos aplicativos voltados para pessoas com TEA. Muitos destes aplicativos focam em atividades específicas, enquanto há aplicativos que visam trabalhar múltiplas habilidades em conjunto e podem ser usados em diferentes contextos (escolar doméstico ou terapêutico). Porém, nem sempre essas ferramentas atendem, de fato, as necessidades dos usuários, levando em consideração suas limitações e anseios. 
O objetivo deste trabalho foi apresentar alguns aplicativos destinados às crianças com TEA para a melhoria da interação do mesmo com o ambiente social em que vivem.

\section{Metodologia}

Esse trabalho buscou apresentar aplicativos e/ou plataformas desenvolvidos para crianças autistas em artigos publicados em periódicos. Para isso, foi feita uma pesquisa bibliográfica, exploratória e descritiva (Gil, 2008).

Foram realizadas buscas nas plataformas "Periódicos Capes", "Google Scholar" e "Web of Science" contendo as palavras: "Autism" e "App" e/ou "Mobile technology". Também foram realizadas buscas de referências cruzadas, nas quais as referências dos artigos encontrados foram analisadas para se ter uma abrangência maior na pesquisa. Buscou-se, na revisão bibliográfica, aplicativos para autistas que apresentam idioma em língua portuguesa, ainda estão disponíveis para download e são preferencialmente gratuitos.

Os aplicativos selecionados nos artigos foram: Neurohab, JADE Autism, Kids ToDo List, Aprendendo com Biel e seus amigos, Matraquinha, ABC Autismo, AutApp, OTO (Olhar, Tocar, Ouvir), TEO Autismo, Autismo Projeto Integrar, Lina Educa, ACA, Brainy Mouse e Livox. Também se procurou, nos artigos, relatos dos benefícios e dificuldades de cada aplicativo.

\section{Resultados e Discussão}

Simões et al. (2014) propuseram uma plataforma denominada Neurohab (Figura 1) para treinamento de atividades diárias, permitindo que cuidadores e terapeutas acompanhem o desempenho e as melhorias dos pacientes com TEA ao longo do tempo. A plataforma possui vários jogos que visam ajudar nas disfunções adaptativas e executivas específicas, desde a higiene pessoal e a manutenção de casa até a identificação monetária, o relacionamento social, a conversação e a exposição ao ar livre. Ele utiliza diferentes tipos de sons: ruídos de animais, ruídos comuns nas ruas, entre outros. Para os cuidadores, uma interface web permite o acesso ao monitoramento das informações do paciente, sua evolução e o uso da plataforma ao longo do tempo. A grande vantagem da plataforma Neurohab, segundo os autores, é que ela pode rodar em diversos dispositivos, como Desktop, Tablets e Smartphones, aumentando as possibilidades de uso da plataforma. Os autores também afirmam que esta plataforma tem potencial de redução dos custos das intervenções e potencializa o acompanhamento dos pacientes. Não foram encontrados resultados de intervenções usando a plataforma.

A plataforma Neurohab está disponível no endereço eletrônico <https://www.neurohab.pt/> e se apresenta nos idiomas português, espanhol e inglês. 
Figura 1. Tela inicial da plataforma Neurohab.

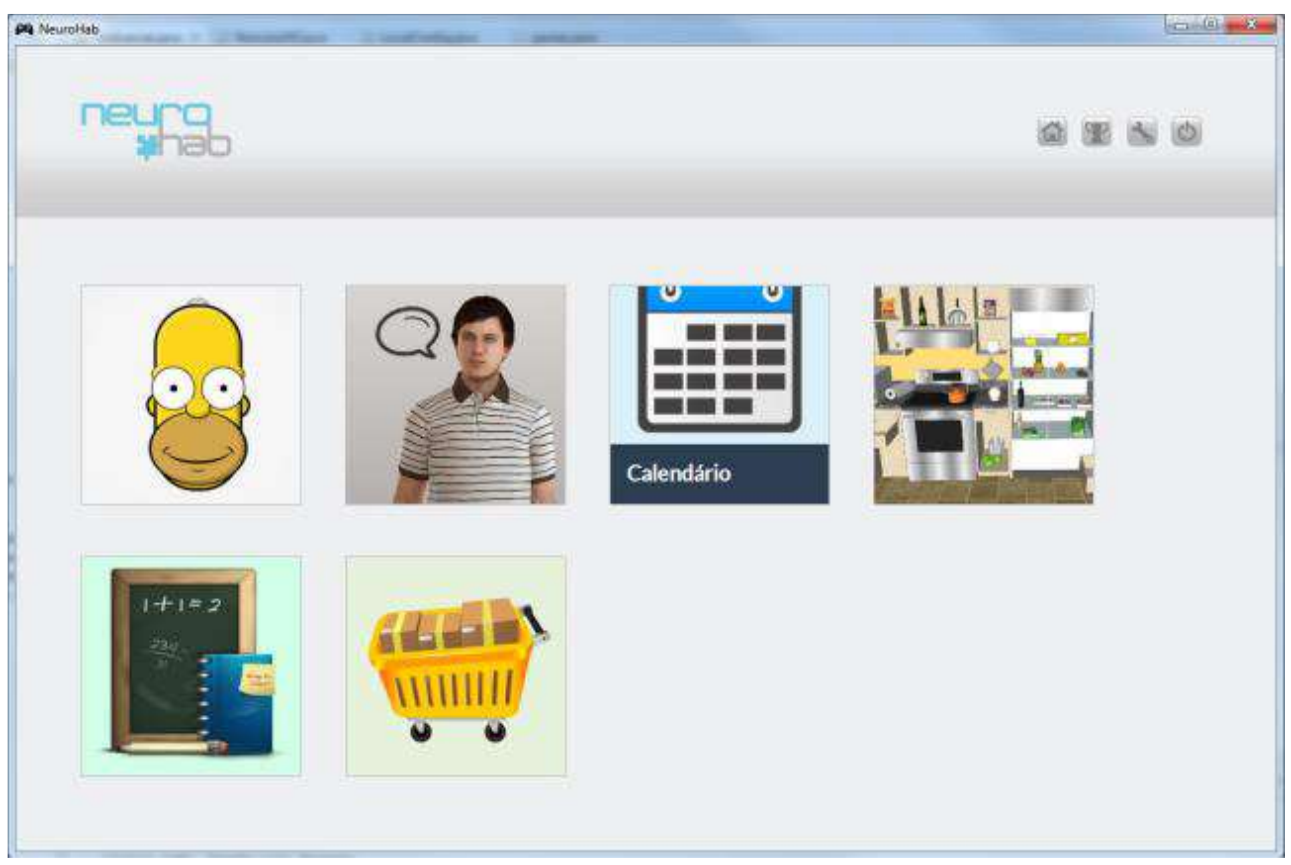

Fonte: Simões et al. (2014).

Segundo Branco, Pinheiro, Damian e Marques (2020), o aplicativo JADE Autism (Figura 2) estimula e desenvolve as funções cognitivas pelo uso de jogos de associação com figuras que fazem parte do dia a dia de toda criança. Essa plataforma utiliza os dados coletados através da interação do paciente com o aplicativo e gera relatórios que apontam algumas de suas dificuldades. Branco et al. (2020) afirmam que esses dados podem auxiliar os terapeutas a traçarem o melhor caminho para o tratamento da criança, o que contribui para que o profissional ajuste o tratamento em um menor tempo, trazendo benefícios, como o desenvolvimento mais rápido e mais adequado da criança.

JADE Autism foi desenvolvido para crianças autistas com base em estudos da ciência da psicomotricidade e dos métodos de desenvolvimento cognitivo. O jogo utiliza equivalência de estímulos e ensino por tentativas discretas e, por meio da ciência de dados, analisa o comportamento do usuário, sendo considerada uma ferramenta de apoio ao tratamento. É um aplicativo que estimula o desenvolvimento cognitivo, a memória, o raciocínio, a habilidade e o desempenho, além de gerar relatórios diante do desempenho da criança ao jogar, apresentando dados valiosos para que os terapeutas envolvidos possam aplicar o melhor plano terapêutico para trabalhar as dificuldades e deficiências apresentadas (Branco et al., 2020).

Branco et al. (2020) apresentaram alguns problemas relatados pelos pais de crianças com TEA em questionários sobre a utilização do aplicativo JADE Autism pelas crianças, como a acessibilidade/interação. Como exemplo, algumas crianças tiveram dificuldades para iniciar o jogo porque precisava inserir o nome do jogador, o que era muito avançado para elas. Outro fato descrito foi a irritação de algumas crianças quando não conseguiam concluir os desafios.

O aplicativo JADE Autism está disponível para acesso livre no endereço eletrônico <http://jadeautism.com/> e na loja Google Play Store (https://play.google.com/store/apps/details?id=com.jadeautism.jadeautism) e apresenta-se em dois idiomas, inglês e português. Alguns elementos interativos e alguns produtos no aplicativo são comprados no jogo, mesmo o aplicativo sendo gratuito. 
Figura 2. Tela do aplicativo JADE Autism.

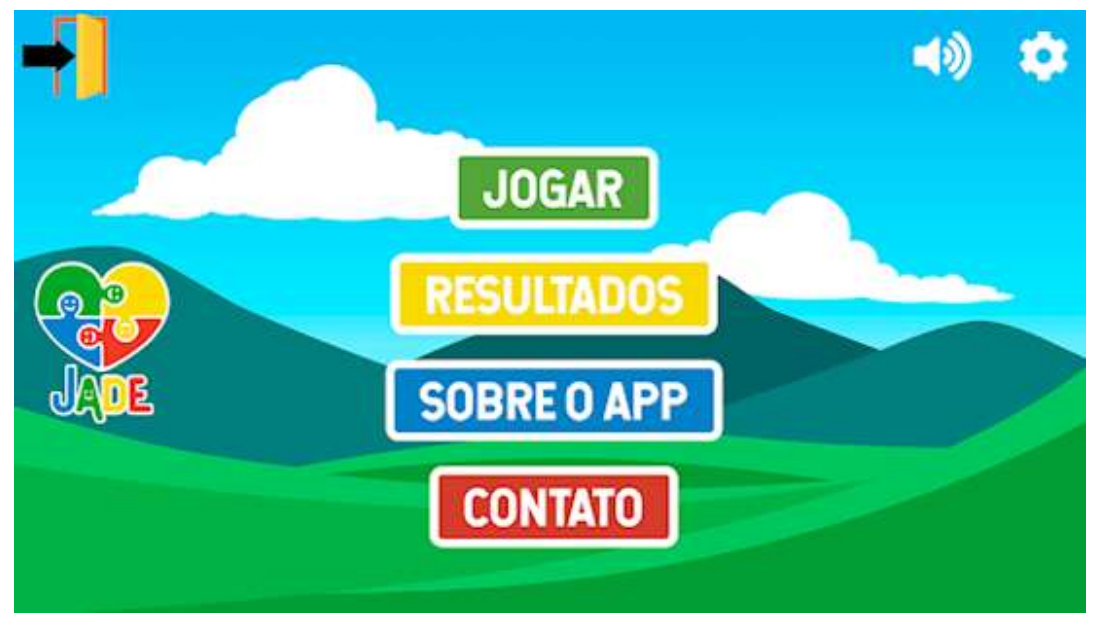

Fonte: Google Play (2021a).

Kids ToDo List (Figura 3) é um aplicativo desenvolvido com base nas atividades normalmente feitas na escola e é indicado para crianças com TEA até 8 anos. Segundo a loja virtual do Google Play (2020), nele existe 100 tipos de cartões que são suportados por sons de voz em vários idiomas, incluindo português (Portugal), sendo que os usuários podem criar cartões de imagens originais adicionando fotos de seus smartphones ou tirando novas com suas câmeras e gravando a própria voz. Os usuários também podem cadastrar a própria programação visual com as tarefas que tiveram de fazer durante o dia fazendo desenhos nos cartões. Neste aplicativo, o usuário recebe uma recompensa quando conclui uma tarefa, como tocar estrelas na tela, coletar peixes, entre outros. A descrição do aplicativo fala que ele é um ótimo cronômetro para as tarefas de rotina diárias das crianças com TEA, como: tomar um banho ou escovar os dentes (Google Play, 2020). Um aspecto negativo dos comentários de usuários que usam o aplicativo Kids ToDo List é que ele possui um relógio, mas não informa o tempo das tarefas, o que seria interessante para disciplinar as crianças com TEA. Outro aspecto negativo é que o aplicativo suporta apenas um perfil de uma criança e pais que possuem dois filhos reclamaram que não conseguem usar dois perfis diferentes no aplicativo. Outro problema que foi relatado é que todos os dias novas tarefas devem ser criadas pois o aplicativo não recarrega as tarefas de forma automática.

O aplicativo Kids ToDo List pode ser acessado na loja Google Play Store (https://play.google.com/store/apps/details?id=jp.co.litalico.kidstodolist) e suporta vários idiomas, inclusive português, devido no aplicativo o usuário poderá criar seus cartões de figuras originais com sua própria foto e gravação de voz. 
Figura 3. Telas do aplicativo Kids ToDo List.

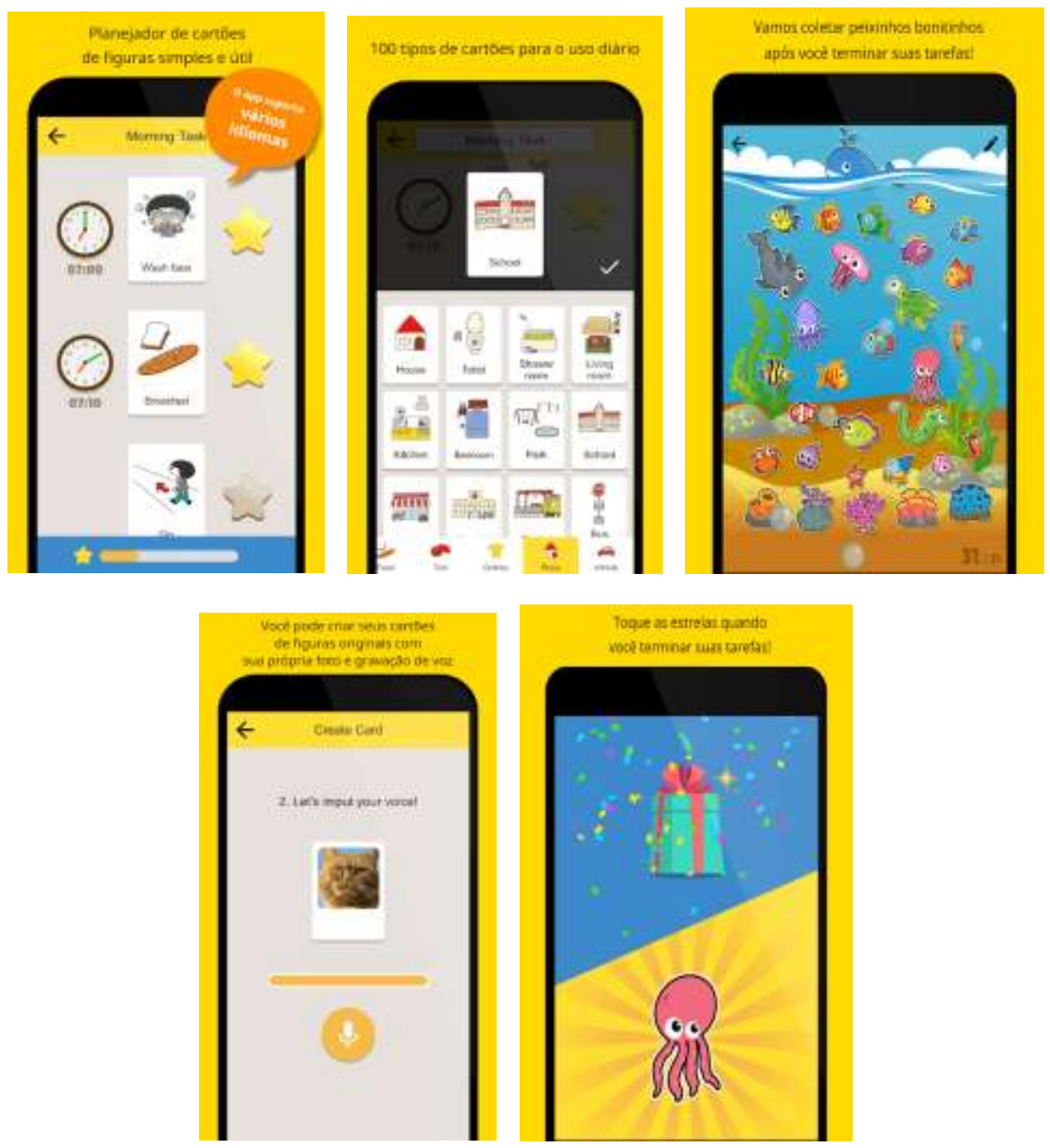

Fonte: Google Play (2020).

O aplicativo Aprendendo com Biel e seus amigos (Figura 4) é indicado para crianças com TEA de faixa etária de dois a oito anos de idade. Ele foi desenvolvido juntamente com os pais de uma criança com TEA e uma equipe com profissionais das áreas de psicopedagogia, fonoaudiologia e terapia ocupacional. Segundo seus desenvolvedores, seu objetivo é ajudar no dia a dia da criança. Foi identificado por Branco et al. (2020), através de questionários aplicados aos pais, que a tela inicial do aplicativo Aprendendo com Biel e seus amigos possui problemas que dificultam a acessibilidade, como muitas animações (incluindo as figuras que se mexem), que podem distrair o usuário, e os métodos de textos na caixa, faltando símbolos que facilitem o usuário identificar o que aquela caixa de texto representa, já que existem crianças com TEA que possuem dificuldades de leitura. Os autores apresentaram relatos de mães que reclamaram que o aplicativo Aprendendo com Biel e seus amigos apresenta poucas variedades nos desafios e não apresenta sons. Em outros relatos de outras mães já foi informado que os recursos variados promoveram boas experiências. Assim, observa-se certa contrariedade nos relatos. 
Figura 4. Telas do aplicativo Aprendendo com Biel e seus amigos.

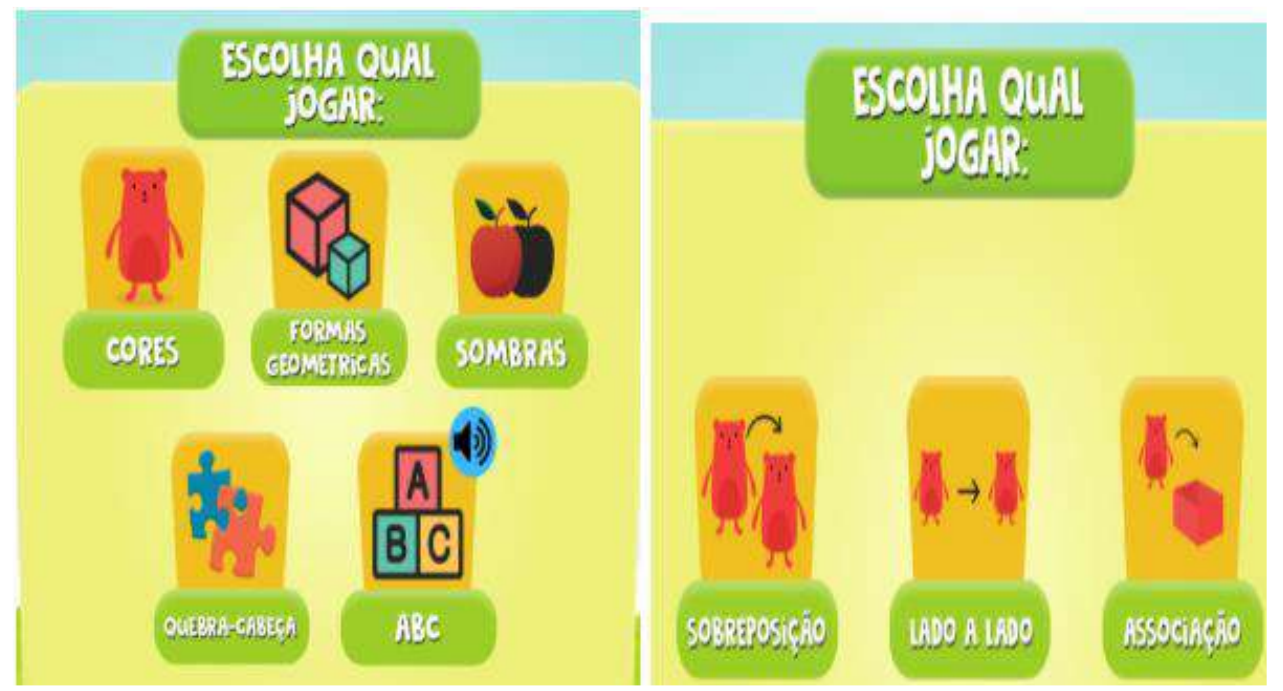

Fonte: Branco et al. (2020).

Outro aplicativo destinado para crianças com TEA, que também foi avaliado por Branco et al. (2020), foi Matraquinha (Figura 5). Nele a criança se comunica através de cartões, com um funcionamento simples: ao clicar nos cartões o aplicativo responde por meio sonoro o que a criança deseja transmitir. No aplicativo existem diversos cartões, desde alguns com as necessidades fisiológicas até outros com atividades de diversão para auxiliar as crianças na comunicação e, ao mesmo tempo, trabalhar a fala dos usuários. Este aplicativo não apresentou relatos negativos dos pais que responderam aos questionários dos autores.

Matraquinha está disponível na Loja Google Play Store (Google Play, 2021b) <https://play.google.com/store/apps/details?id=com.phonegap.matraquinha> e também no sítio $<$ https://www.matraquinha.com.br/>, sendo disponível gratuitamente e em língua portuguesa.

Figura 5. Telas do aplicativo Matraquinha.

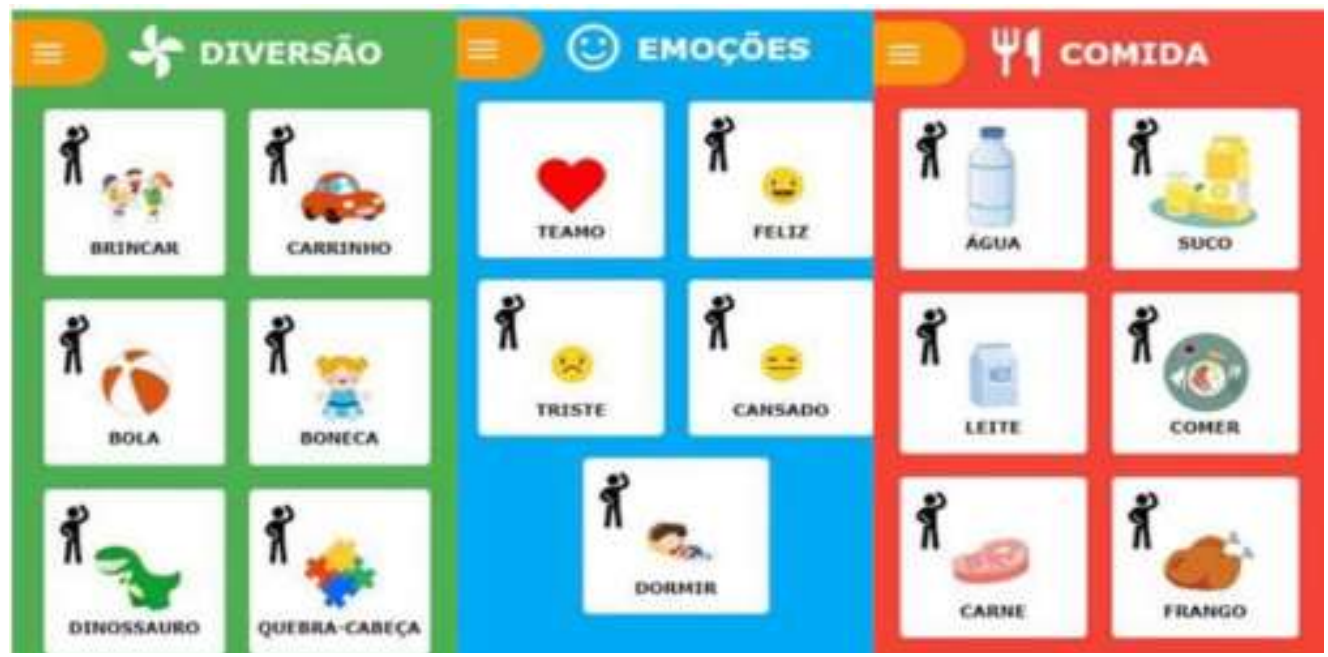

Fonte: Branco et al. (2020).

O aplicativo ABC Autismo (Figura 6) foi desenvolvido por pesquisadores do Instituto Federal de Alagoas (IFAL) com o objetivo de auxiliar no processo de alfabetização e servir como ferramenta de apoio no tratamento e na educação de crianças 
e adolescentes com TEA. O aplicativo apresenta 40 fases interativas distribuídas em quatro níveis de dificuldade. Diversas características foram implementadas e, segundo os autores, pode-se destacar, entre elas, a ordem crescente de nível e a diferenciação entre tamanhos, formas e cores dos objetos representados (Farias; Silva, \& Cunha, 2014; Aragão et al., 2019).

Figura 6. Telas dos quatro níveis do aplicativo ABC Autismo.

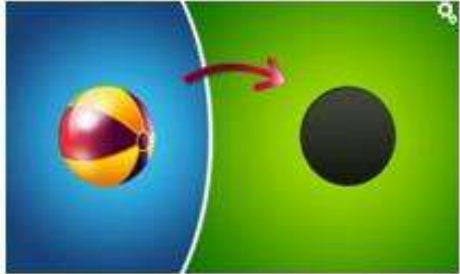

Nivel 1

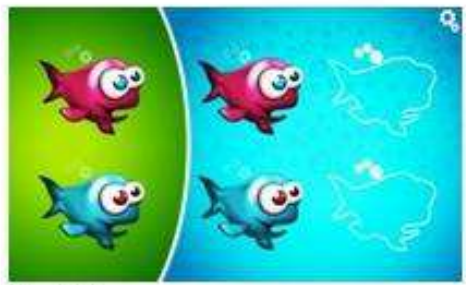

Nivel 3

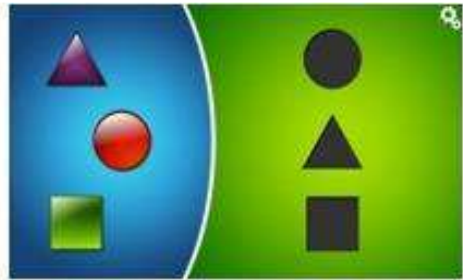

Nivel 2

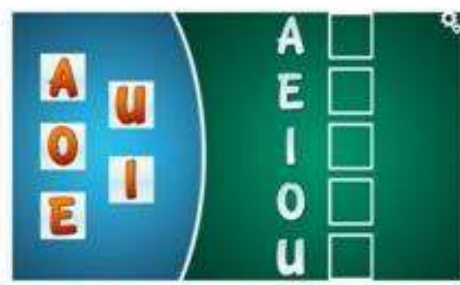

Nivel 4

Fonte: Aragão et al. (2019).

Em questionários para avaliar o aplicativo ABC Autismo, também aplicados por Branco et al. (2020) aos pais de crianças com TEA, os relatos indicaram boas experiências em crianças com TEA, sendo ligadas aos desafios, cores e sons do aplicativo. Entretanto, resultados negativos foram encontrados devido às dificuldades encontradas pelas crianças. Ocorreram relatos que as crianças demonstraram irritação quando não conseguiam concluir os desafios do aplicativo. Outro relato negativo foi de que nos últimos níveis de dificuldade ocorre muito travamento nos jogos do aplicativo. Lucian e Stumpf (2019) também avaliaram o aplicativo e relataram que dificuldades foram encontradas durante o processo de interação com o aplicativo ABC Autismo, no nível 4, por ser composto de tarefas alfabetizadoras em que possuem composição de palavras, letramento, sequenciamento de números, entre outros. A dificuldade de interação foi percebida pela falta de recursos de interação de comando de voz para capacitar o reconhecimento de letras e números, como também para o aperfeiçoamento da comunicação, fato que pode restringir a funcionalidade do aplicativo e o atendimento às expectativas dos usuários. Barbosa $e t$ al. (2020), que também avaliaram o aplicativo ABC Autismo, identificaram um ponto negativo por possuir vários sons altos e desnecessários que são acionados quando se muda de fase ou faz uma interação errada, não levando em conta a sensibilidade auditiva de crianças com autismo. Porém, foi destacado, como ponto positivo, uma versão recente específica para animais com bastante imagens interativas.

O AutApp (Figura 7) é um aplicativo móvel com atividades que buscam auxiliar as crianças com TEA de uma maneira divertida. Foi desenvolvido por Gabriel Hahn Schaeffer, em 2017, e possui várias fases para auxiliar a coordenação motora da criança e o reconhecimento de formas e cores. O aplicativo incentiva a criança a reconhecer as emoções do personagem Erick, no qual ela pode escolher através de opções ou tirar uma foto imitando o personagem. Entretanto, a foto da criança não é salva em nenhum local, nem mesmo no dispositivo. É necessário que haja acesso à internet na opção com foto para que a tecnologia faça o reconhecimento da expressão facial. O aplicativo está bem avaliado na loja de aplicativos, mas foram encontradas reclamações sobre a baixa quantidade de atividades e a ausência de sons para auxiliar as crianças a se expressarem (Google Play, 2017). 
O aplicativo AutApp está disponível na loja Google Play Store no endereço eletrônico <https://play.google.com/store/apps/details?id=io.gabriel.hahn.autapp> e apresenta-se no idioma português.

Figura 7. Tela inicial do aplicativo AutApp.

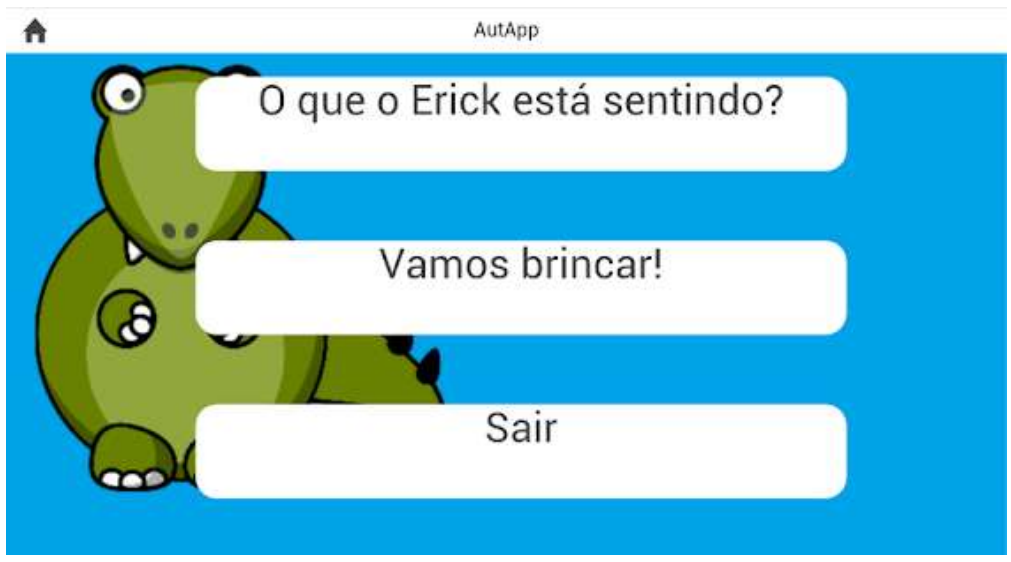

Fonte: Google Play (2017).

O aplicativo OTO - Olhar Tocar Ouvir (Figura 8) foi desenvolvido por Rodrigues e Abilhoa (2015) para o aprendizado do alfabeto por crianças com TEA. Ele usa imagens e sons associados com as respectivas letras do alfabeto, como demonstrado na Figura 9, em que a letra A relaciona-se ao nome ABELHA, a letra B com BALEIA, C com a palavra CASTOR, a letra D com DINOSSAURO, entre outros. Pais que utilizaram este aplicativo com seus filhos demonstraram satisfação em relação ao auxílio que o OTO teve em ajudar na fala e na alfabetização das crianças, pois eles melhoraram seus rendimentos e interações escolares. Alguns pais apresentaram relatos negativos na utilização do aplicativo nas respostas ao questionário aplicado por Branco et al. (2020), no qual se mostraram insatisfeitos devido a pouca variedade de desafios do aplicativo. Este aplicativo está disponível gratuitamente para smartphones e tablets com sistema operacional Android na loja Google Play <https://play.google.com/store/apps/details?id=br.com.myalphabeto〉.

Figura 8. Tela inicial do aplicativo OTO - Olhar Tocar Ouvir.

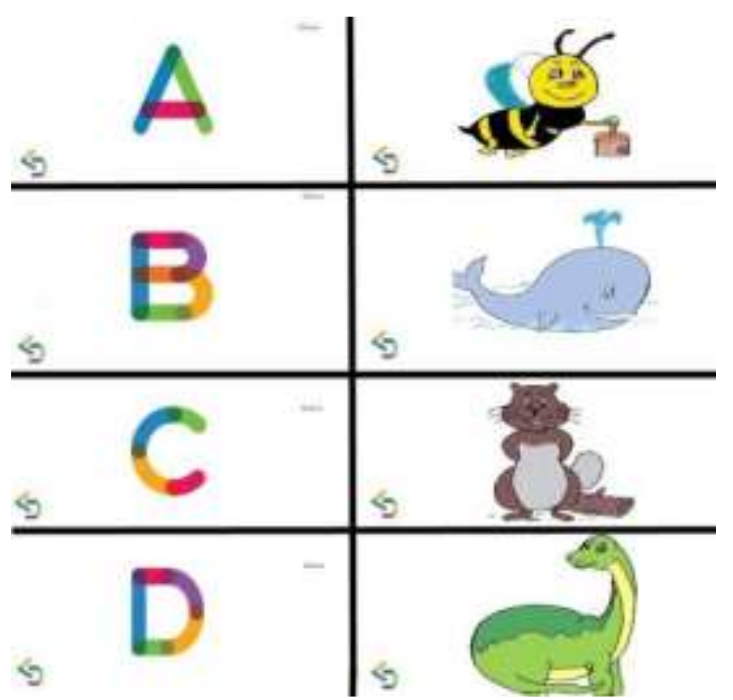

Fonte: Rodrigues e Abilhoa (2015). 
O aplicativo TEO Autismo (Tratar, Estimular, Orientar) (Figura 9) foi desenvolvido por pesquisadores e estudantes do curso de Ciência da Computação da Universidade Federal de Alagoas - Campus Arapiraca, trazendo jogos interativos voltados para dispositivos móveis para auxiliar no tratamento de crianças com TEA (Lucian \& Stumpf, 2019).

Esse aplicativo é divido em níveis, ocasionando o aprofundamento da aprendizagem do usuário à medida que a dificuldade exigida pelo aplicativo aumenta. Ele possui uma variedade de ícones e cores que auxiliam na navegação entre as telas, deixando o aplicativo intuitivo e dinâmico. O feedback acontece de forma imediata, utilizando sons e efeitos de comemoração quando se atinge o objetivo do jogo, ajudando o usuário a visualizar os resultados de suas tarefas. $\mathrm{O}$ aplicativo traz a possibilidade de obtenção de relatórios de progresso das crianças, o que ajuda no acompanhamento. Um ponto negativo do aplicativo é a sua monotonia, mesmo tendo grande variedade de atividades para o aprendizado, o que o deixa cansativo (Lucian \& Stumpf, 2019).

Figura 9. Tela inicial do aplicativo TEO Autismo

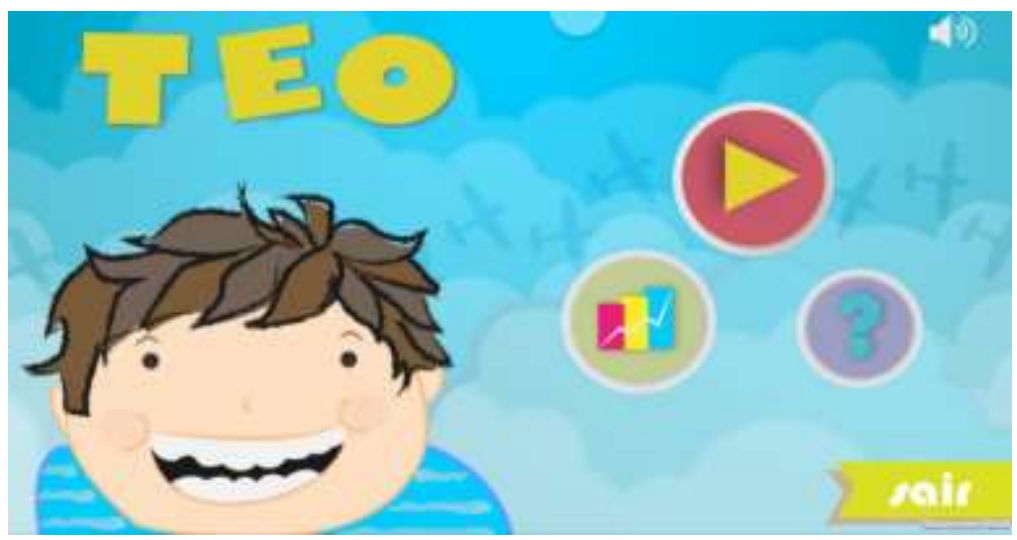

Fonte: Lucian e Stumpf (2019).

O aplicativo Autismo Projeto Integrar (Figura 10) é resultado da pesquisa de conclusão dos cursos de Especialização em Desenvolvimento de Software para Internet e Especialização em Tecnologias da Informação e Comunicação da Universidade Federal do Acre e foi projetado para celulares e tablets que utilizam sistema Android. Ele pode ser baixado

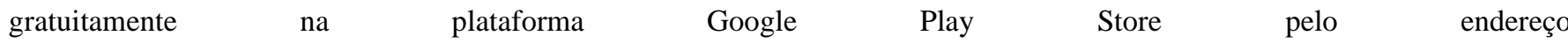
<https://play.google.com/store/apps/details?id=poder.ufac.br.autismoprojetointegrar> (Lucian \& Stumpf, 2019).

O objetivo do aplicativo Autismo Projeto Integrar é auxiliar pessoas com Transtorno do Espectro Autista (TEA) na organização de suas atividades da vida diária através do apoio audiovisual dos desenhos roteirizados do site Autismo Projeto Integrar <http://autismoprojetointegrar.com.br/> sobre comportamento, higiene e uso do banheiro. $\mathrm{O}$ aplicativo permite também o cadastro do mural "Meus Compromissos" com até 18 atividades representadas em desenhos roteirizados que indicam o passo a passo de como será a rotina da pessoa com TEA, exibindo uma agenda com as atividades do dia. Embora o aplicativo Autismo Projeto Integrar se intitule jogo, ele não possui muitas características de jogo e sua interface é o único ponto que se assemelha a um recurso de jogo, pois elas são bem coloridas e variadas. 
Figura 10. Tela inicial do aplicativo Autismo Projeto Integrar

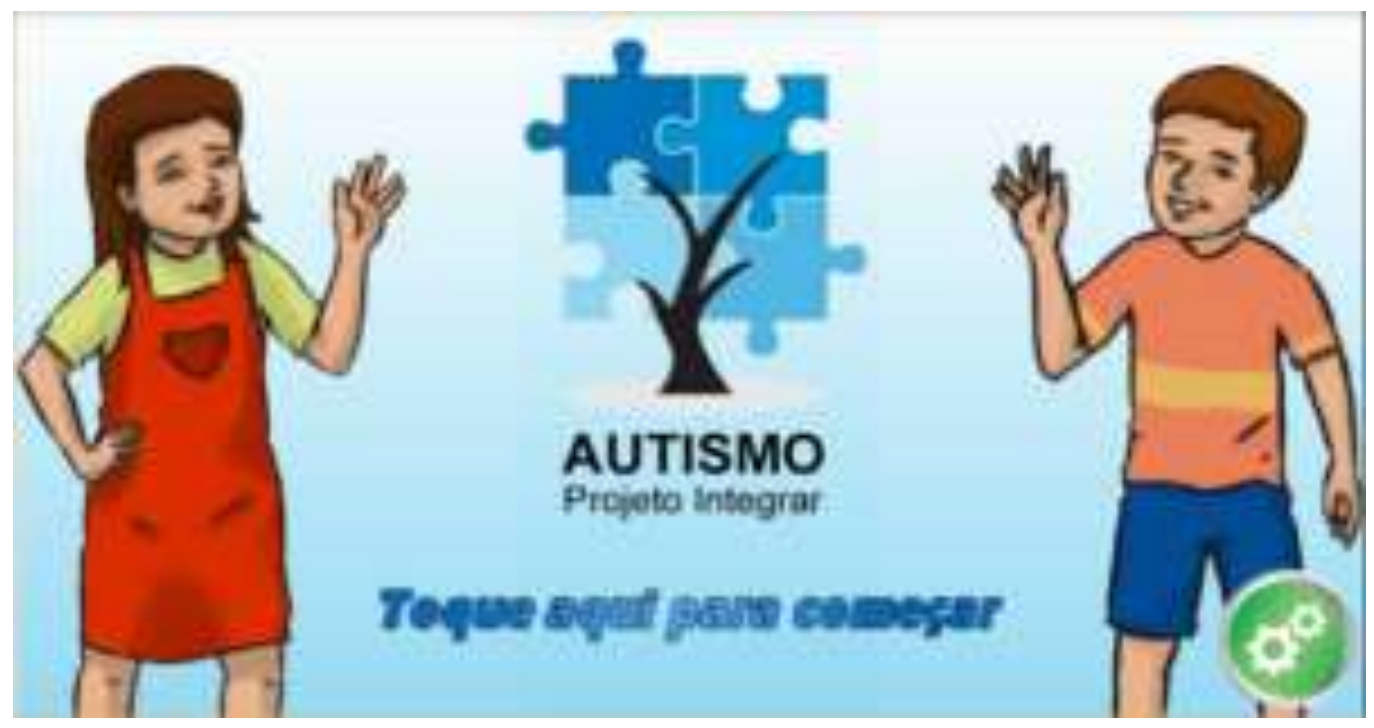

Fonte: Lucian e Stumpf (2019).

O aplicativo Lina Educa (Figura 11) foi desenvolvido por pesquisadores da Universidade Federal do Amazonas (UFAM) e é destinado ao reforço ou apoio às atividades educacionais de crianças com TEA, não sendo utilizado como único ou principal recurso de educação para crianças autistas. Para realizar as atividades de alfabetização, a criança com TEA deverá obrigatoriamente possuir algumas habilidades desenvolvidas, tais como: interesse por letras, palavras e livros; responder mediante a estímulos; discriminação espacial (posição); discriminação de quantidade (por exemplo: mais x menos); e discriminação das dimensões críticas dos estímulos textuais, por aspectos gráficos e sonoros. As atividades para o desenvolvimento da escrita não são estimuladas pelo Lina Educa e elas deverão ser conduzidas e planejadas mediante a supervisão de um especialista (Gomes \& Silva, 2007).

O Lina Educa possui dois ambientes virtuais: um destinado ao educador e outro à criança com TEA. O ambiente do educador permite a elaboração da agenda semanal da criança com seleção tanto das atividades de alfabetização quanto das atividades da vida diária. Esse ambiente é protegido por senha e não permite o acesso pela criança. $\mathrm{O}$ ambiente da criança não é protegido por senha e o acesso é feito por meio de um ícone com foto da criança quando o aplicativo é utilizado por mais de um usuário. Este ambiente mostra apenas as atividades selecionadas pelo educador (Gomes \& Silva, 2007).

O download deste aplicativo pode ser realizado no sítio <http://www.linaeduca.com/> de forma gratuita e em língua portuguesa. 
Figura 11. Tela do aplicativo Lina Educa.

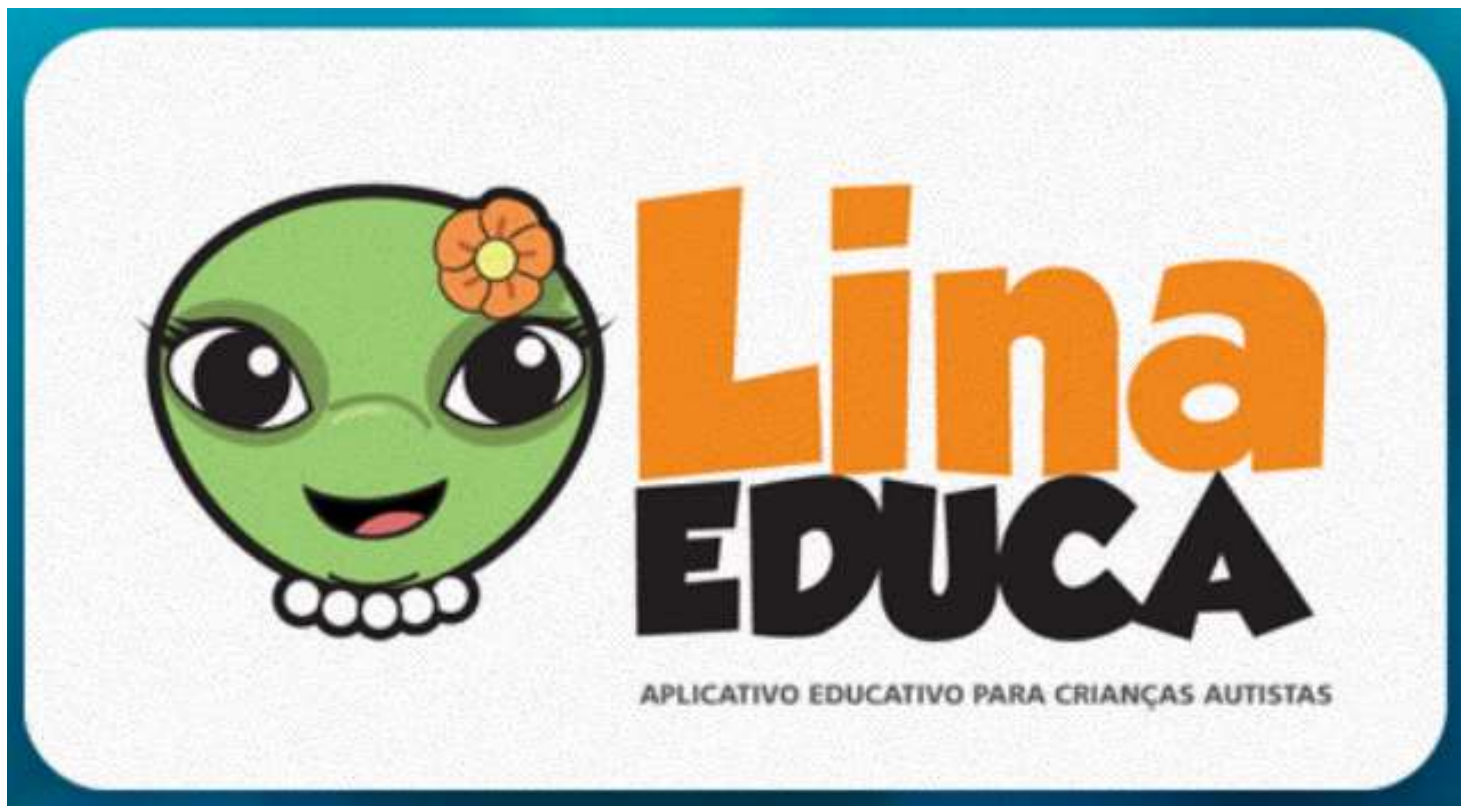

Fonte: Gomes e Silva (2007).

Gobbo, Barbosa, Morandini, Mafort e Mioni (2018) desenvolveram o aplicativo ACA - Aprendendo com Comunicação Alternativa (Figura 12) para crianças com TEA de crianças/adolescentes de 3 a 15 anos visando o ensino destas crianças para ajudar no vocabulário e auxílio à alfabetização. Resultados mostraram que os participantes da pesquisa tiveram ganhos no vocabulário. Em relação ao auxílio da alfabetização, os que conseguiram concluir 100\% dos níveis já estavam alfabetizados, porém os autores acreditam que a prática pode potencializar o entendimento dessas crianças em relacionar imagens com suas atividades da vida diária, já que o aplicativo foi desenvolvido com a utilização de imagens para o ensino do alfabeto, sílabas e palavras. Um ponto negativo encontrado foi a grande quantidade de informações contidas na tela e/ou imagens, prejudicando a atenção das crianças com TEA ao utilizarem este aplicativo, o que dificultou maior atenção para finalização das tarefas. 
Figura 12. Tela do aplicativo $A C A$.

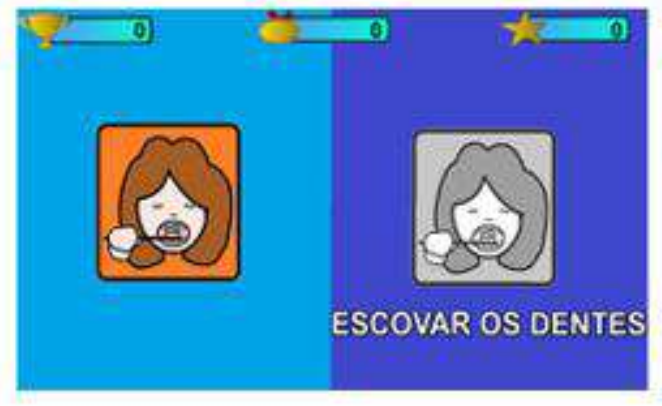

a

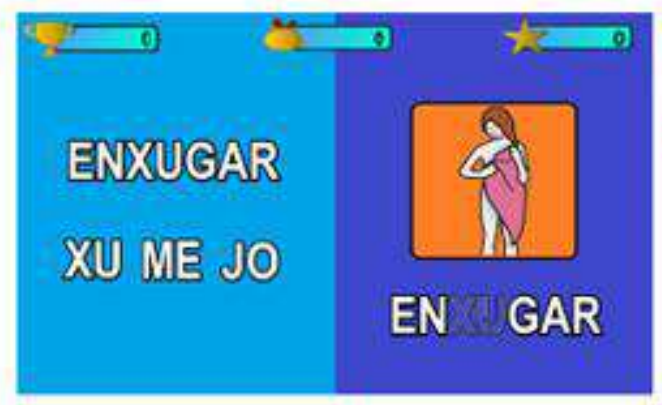

c

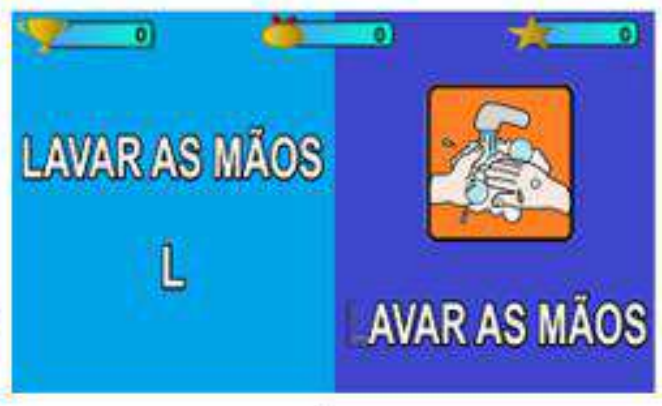

b

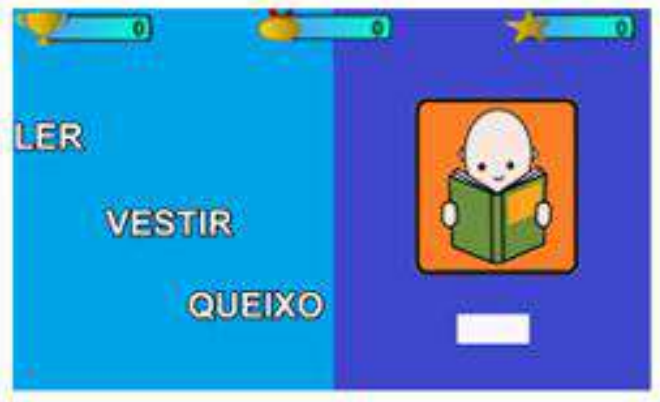

d

Fonte: Gobbo et al. (2018).

O aplicativo Brainy Mouse (Figura 13) foi desenvolvido para crianças com TEA para ajudá-las na alfabetização. Ele conduz o processo com a história de um ratinho em um restaurante e utiliza cenários de cozinhas de oito países diferentes, dentre eles, o Brasil. A criança precisa pegar a comida para fazer uma receita e durante o percurso ela forma as palavras, prestando atenção às cores, associadas a sons e gráficos. Essa interação aconteça de forma lúdica e o aplicativo permite que as crianças com TEA customizem o ratinho, protagonista da história, além de serem desafiadas a juntar moedas virtuais (denominadas cheese coin) (Barbosa et al., 2020).

Este aplicativo possui um canal no Youtube com vários vídeos ensinando a sua utilização, disponível no endereço eletrônico <https://www.youtube.com/channel/UCO8xvT1QBwaUJXA9C08ivtQ/featured>. 
Figura 13. Tela do aplicativo Brainy Mouse.

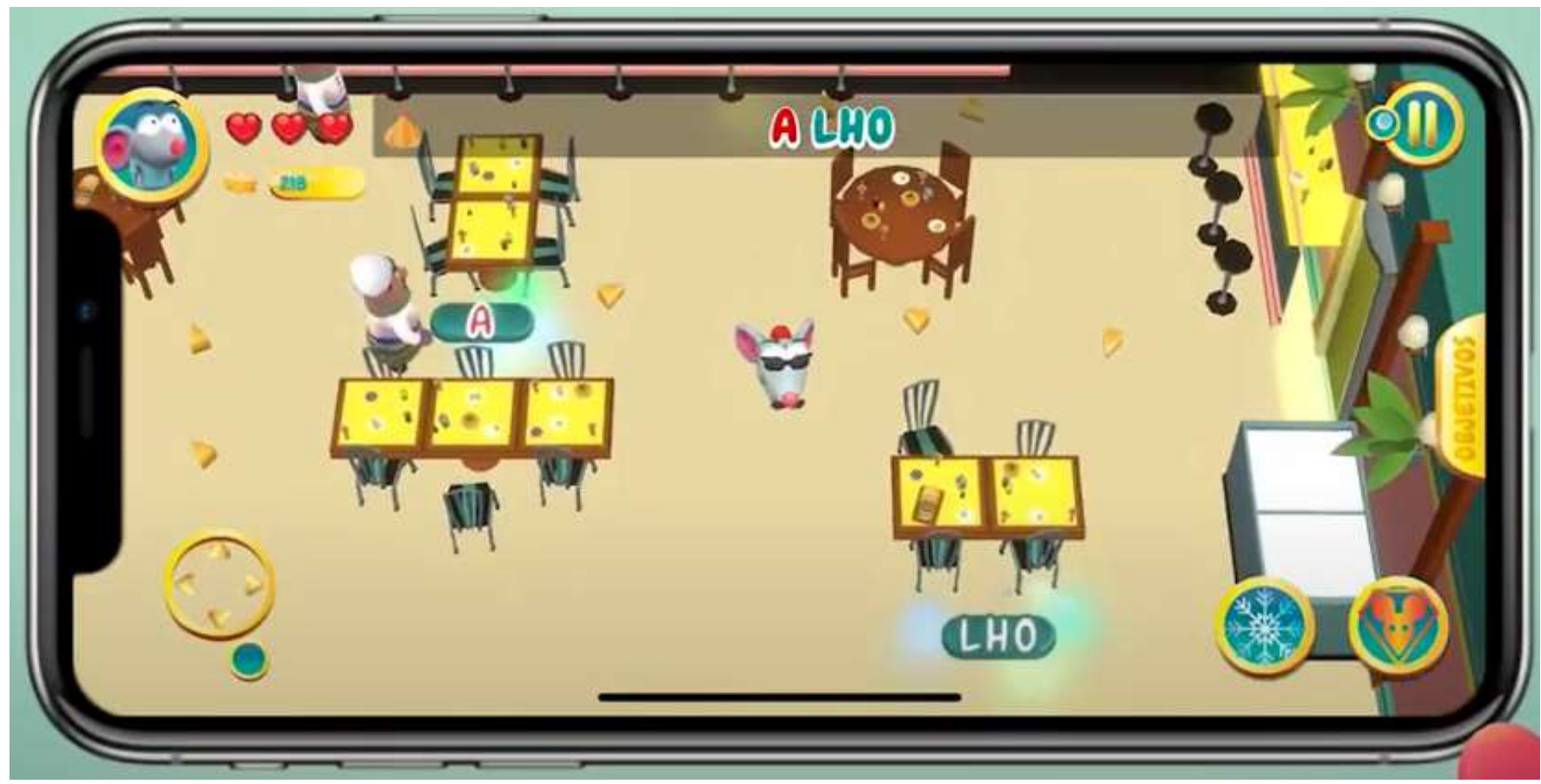

Fonte: Brainy Mouse Foundation, (2018).

O aplicativo Livox (Figura 14) está disponibilizado de forma gratuita para download na loja do Google Play (Google Play, 2021c) <https://play.google.com/store/search?q=livox\%20app\&c=apps>. Nas informações do aplicativo, na própria loja, é informado que é um aplicativo pago e que necessita da aquisição do direito de uso de uma licença. Ele foi vencedor de um prêmio da ONU (Organizações das Nações Unidas) de melhor aplicativo de inclusão. O Livox (Liberdade em voz alta) foi criado pelo analista de sistemas pernambucano Carlos Pereira, pai de uma menina com paralisia cerebral, chamada Clara, para que ela pudesse se comunicar com seus pais. Este aplicativo foi traduzido para 25 idiomas e é o único no mundo a utilizar inteligência artificial, aprendizado de máquina e processamento natural de linguagem para levar formas de comunicação, interação e aprendizado para o usuário. É, também, um portal de gestão e avaliação, no qual é possível gerir o uso da ferramenta e avaliar periodicamente a evolução de cada paciente. O Livox corrige o toque imperfeito, funcionando com o piscar dos olhos através de algoritmos reconhecidos.

O Livox também é uma loja de conteúdos onde os profissionais e famílias que se beneficiam do uso e compartilham seus conteúdos (pranchas de comunicação ou ensino) com os demais usuários do Livox do mundo. Este aplicativo têm a opção de uma assinatura mensal ou de licenças vitalícias, de acordo com a versão escolhida do software, e também é disponível para governos (grandes volumes).

Algumas mães que utilizaram o Livox emitiram comentários negativos com relação à sua versão gratuita, que é muito simples. Outros comentários solicitavam uma avaliação da versão paga do aplicativo por 30 dias de forma gratuita e/ou o patrocínio de órgãos públicos para utilização por crianças com deficiências (Oliveira, 2017; Livox, 2021). 
Figura 14. Tela do aplicativo Livox.

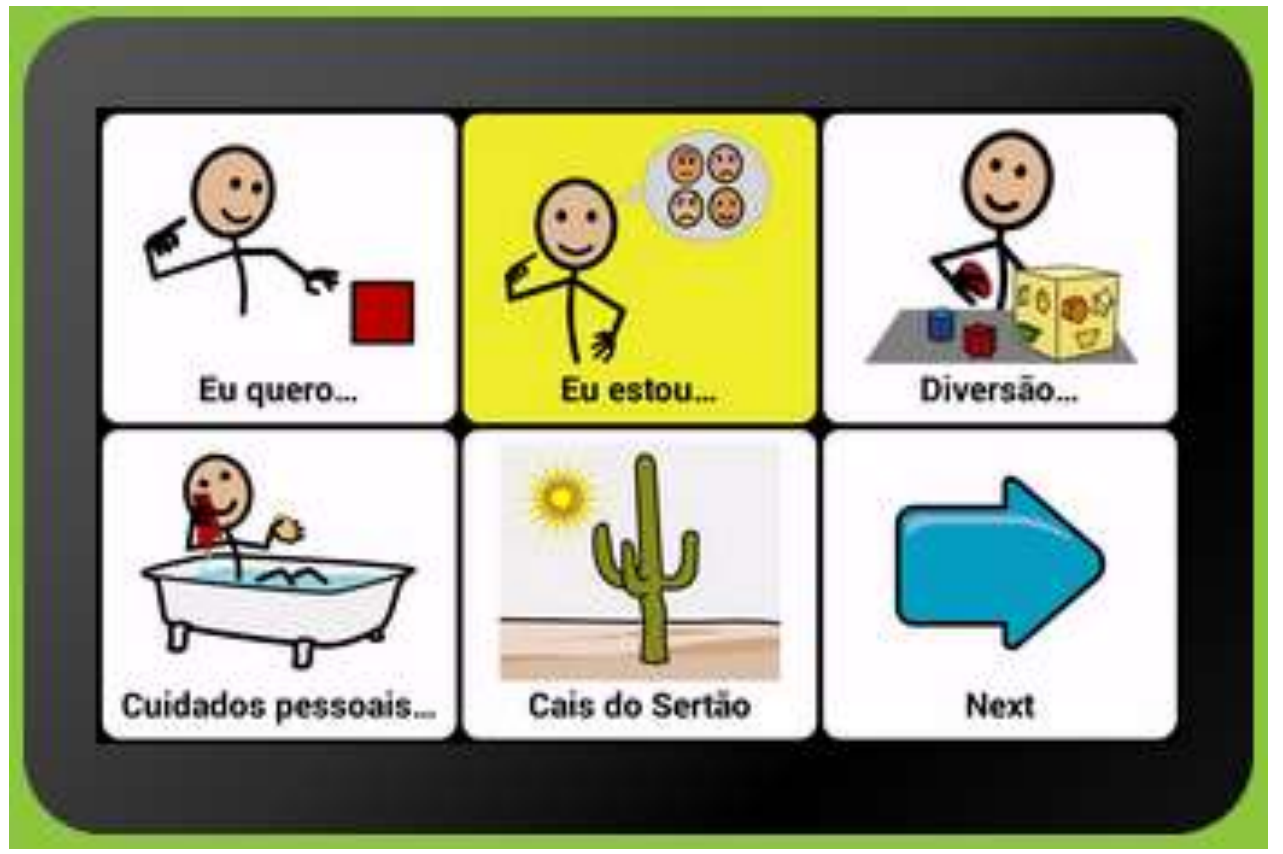

Fonte: Livox (2021).

\section{Considerações Finais}

Todos os aplicativos citados neste trabalho estão disponíveis para download e, com exceção de um que possui planos pagos para a utilização de níveis mais avançados, são totalmente gratuitos, o que auxilia a reduzir os custos do atendimento clínico às crianças com TEA.

A maioria dos aplicativos descritos nesta revisão utiliza imagens reais relacionadas ao dia a dia de crianças em ambientes de seu convívio, como escolares e domiciliares, e objetiva facilitar a comunicação das crianças com os seus responsáveis, ajudando-as a escolherem o que desejam e a se expressarem. Os aplicativos também costumam apresentar sons referentes às imagens e às ações, o que pode auxiliar as crianças no desenvolvimento da fala.

Os aplicativos que apresentaram atividades interativas, recursos de som, uso de imagens e animações adequadas ao aprendizado de pessoas com autismo foram os que tiveram avaliações mais positivas quanto ao desenvolvimento de crianças com TEA. Aplicativos que requereram textos, como a escrita de nomes para o seu início, apresentaram reclamações pois várias crianças não tinham capacidade de entenderem o que estava sendo pedido e inserirem os nomes, sendo isso realizado pelos responsáveis. Aplicativos que continham histórias cujos roteiros deveriam ser preparados pelas crianças ou pelos responsáveis também foram avaliados negativamente.

Observou-se que, em geral, os usuários desejam aplicativos com grande quantidade e variedade de atividades, além de imagens representativas com sons e uso de gamificação de forma a se aprofundar os níveis e fornecer recompensas aos usuários de forma a incentivá-los. Os relatos dos responsáveis pelas crianças também demonstraram a necessidade de melhoria dos aplicativos existentes ou de criação de novos aplicativos. Espera-se que este artigo também seja útil para desenvolvedores de aplicativos de forma que eles consigam planejar os aplicativos de acordo com o que os usuários desejam.

Sugere-se, para trabalhos futuros, que os aplicativos listados sejam testados em um maior número de usuários para que se verifique, com maior profundidade, os seus pontos fortes e fracos. Dessa forma, os aplicativos poderão ser melhorados 
ou novos aplicativos poderão ser produzidos considerando-se esses pontos, de forma a auxiliar ainda mais a melhorar a qualidade de vida das famílias que possuem pessoas com TEA.

\section{Referências}

Aragão, M., Bottentuit Junior, J., \& Zaqueu, L. (2019). O uso de aplicativos para auxiliar no desenvolvimento de crianças com TEA. Revista Olhares $e$ Trilhas, 21(1). Recuperado em 26 maio, 2021, de http://www.seer.ufu.br/index.php/olharesetrilhas/article/view/46088.

Barbosa, C.R.S.C., Artoni, A.A\&., Felinto, A.S. (2020, november). Jogos educativos para crianças com transtorno do espectro autista: auxílio na comunicação e alfabetização. Proceedings of $19^{\circ}$ Computer Games and Digital Entertainment (SBGames 20), Recife, PE, Brasil, $663-672$.

Bölte, S., Golan, O., Goodwin, M.S, \& Waigenbaum, Z.L. (2010). What can innovative technologies do for Autism Spectrum Disorders? SAGE Publications and The National Autistic Society, 14(3), 155-159.

Branco, K.S.C., Pinheiro, V.M.S., Damian, A.L., Marques, A.B.S. (2020). Investigating the first user experience and accessibility of educational applications for autistic children. Anais do XIX Simpósio Brasileiro Sobre Fatores Humanos Em Sistemas Computacionais. Porto Alegre: Sociedade Brasileira de Computação.

Brasil. Lei $n^{o}$ 12.764, de 27 de dezembro de 2012 (2012). Institui a política nacional de proteção dos direitos das pessoas com transtorno do espectro autista. Brasília, 2012. Recuperado em16 julho, 2021, de http://www.planalto.gov.br/ccivil_03/_ato2011-2014/2012/lei/112764.htm

Brainy Mouse Foundation. Brainy Mouse Foundation - O jogo Brainy Mouse. Youtube, 12 abr. 2018. Recuperado em 19 de julho, 2021, de https://www.youtube.com/channel/UCO8xvT1QBwaUJXA9C08ivtQ/featured

Farias, E.B., Silva, L.W.C., Cunha, M.X.C. (2014, maio). ABC AUTISMO: Um aplicativo móvel para auxiliar na alfabetização de crianças com autismo baseado no Programa TEACCH. In: Anais do X Simpósio Brasileiro de Sistemas de Informação, Londrina, PR, Brasil, $458-469$.

Gil, A.C. 2008. Como elaborar projetos de pesquisa. 4. ed. São Paulo: Atlas.

Gobbo, M.R.M., Barbosa, C.R.S.C., Morandini, M., Mafort, F., \& Mioni, J.L.V.M. (2018, october/november). Jogo ACA para indivíduos com Transtorno do Espectro Autista. Proceedings of $17^{\circ}$ Computer Games and Digital Entertainment (SBGames 18), Foz do Iguaçu, PR, Brasil, $1114-1121$.

Gomes, A.N., \& Silva, C.B. (2007, october). Educational Software for Autistic Children of Severe Level. In: 4th International Conference on Design Research, Rio de Janeiro, RJ, Brasil. Recuperado em 13 de julho, 2021, de: http://www.linaeduca.com/artigo/software-para-autismo-Alice_NevesClaudete_Barbosa.pdf.

Google Play. (2017). AutApp. Recuperado em 03 de julho, 2021, de https://play.google.com/store/apps/details?id=io.gabriel.hahn.autapp

Google Play. (2020). Kids ToDo List. Recuperado em 28 de junho, 2021, de https://play.google.com/store/apps/details?id=jp.co.litalico.kidstodolist

Google Play. (2021a). Jade Autism. Recuperado em $\quad 2021, \quad$ de https://play.google.com/store/apps/details?id=com.jadeautism.jadeautism\&hl=pt_BR\&gl=US

Google Play. (2021b). Matraquinha. Recuperado em 17 de julho, 2021, de https://play.google.com/store/apps/details?id=com.phonegap.matraquinha

Google Play. (2021c). Livox. Recuperado em 15 de julho, 2021, de https://play.google.com/store/apps/details?id=br.com.livox

Livox. (2021). Recuperado em 19 de julho, 2021, em https://livox.com.br/br/

Lucian, B.O., \& Stumpf, A. (2019) Análise de aplicativos destinados ao aprendizado de crianças com transtorno do Espectro Autista. Revista Design \& Tecnologia, 9(19), 43-65.

Oliveira, K.S. (2017). VoxLaPS: um aplicativo gratuito de comunicação aumentativa e alternativa. Dissertação (Mestrado em Ciência da Computação). Universidade Federal do Pará- UFPA. Belém, PA.

Rodrigues, J.H., \& Abilhoa, A.C.E.S.L. (2015). OTO: Um Aplicativo Android para Auxílio da Aprendizagem de Crianças Portadoras de Transtorno do Espectro Autista. Trabalho de Conclusão de Curso (Graduação em Análise e Desenvolvimento de Sistemas), Faculdade Guairacá, Guarapuava, PR, Brasil.

Silva, M. D. da, Oliveira, M. da C., Campos, C. de S., \& Oliveira, E. N. A. de. (2019). The playful from games and play in inclusive teaching of children with autistic spectrum disorder (ASD): a literature review. Research, Society and Development, 8(4), e1084943.

Silva, J. P. da, \& Silva, P. J. da. (2016). Students with autism in the regular classroom: what to do?. Research, Society and Development, 2(2), 122-135.

Simões M.; Mouga, S.; Pedrosa, F.; Carvalho, P.; Oliveira, G., \& Branco, M.C. (2014). Neurohab: a platform for virtual training of daily living skills in autism spectrum disorder. Procedia Technology, 16, 1417-1423.

Soomro, N.; Soomro, S. (2018). Autism Children's App using PECS. Annals of Emerging Technologies in Computing. 2(1), 7-16.

Souza, R.F.S., Silva, R.I.P., \& Silva, N.A.S. (2019). O processo de inclusão do aluno autista: desafios à prática docente. In: M.B. Justus (Org.). Políticas públicas na educação brasileira: caminhos para a inclusão 2. Ponta Grossa, PR: Atena. 